\title{
The influence of prescriptive and subjective phrase markers on retrieval latencies
}

\author{
MICHAEL L. HILLINGER, CARLTON T. JAMES, DEBORAH L. ZELL, and LAURA M. PRATO \\ Rutgers University, New Brunswick, New Jersey 08903
}

\begin{abstract}
To investigate the effect of subjective (pause defined) and prescriptive (grammatically defined) phrase markers on subjective grouping, subjects were presented simple declarative sentences in which the subjective and prescriptive phrase markers were either the same or different. In the same condition a 2 -sec pause was placed at the major syntactic boundary, while in the different condition the pause was inserted within a constituent. Retrieval latencies for words following probes in the sentence were influenced by grammatical structure and, to a lesser extent, by the pause. The results suggest that, while sentential organization may be influenced by inserting pauses, this influence is highly constrained by the sentence's grammatical structure.
\end{abstract}

Previous studies concerned with the effect of subjective organization on retrieval of linguistic material fall into two categories: Some have imposed an external grouping structure by the introduction of pauses or by the spatial grouping of list elements (Bower \& Winzenz, 1969; DeRosa \& Baumgarte, 1971; McLean \& Gregg, 1967; Wilkes \& Kennedy, 1969; Winzenz \& Bower, 1970); others have allowed subjects the liberty to impose their own subjective organization on the series (Butterfield \& Belmont, 1971; Martin, 1970). Most studies have employed a retrieval task from which subjective organization is inferred from the pattern of retrieval latencies during ordered or probed recall. The retrieval task, based on Sternberg's (1969) proberecall technique, consists of presenting a set of to-be-remembered items, followed by a probe item from that set. When a probe is shown, subjects are required to give the item that followed the probe in the memorized set. Using auditory or visual pausing as a means of marking structure, a positive correlation has been found between the pausing strategy adopted and the retrieval latency. Such studies have demonstrated that for strings of unrelated items, the temporal grouping of the sequence determined the retrieval units, thereby imposing a subjective structure on serial lists which influences encoding and retrieval strategies.

One may question whether this finding would hold true for more organized materials, such as sentences. Serial lists or strings composed of unrelated items have no inherent grammatical structure that would influence subjective organization for acquisition and retrieval. Therefore, experimenter-imposed pauses are easily adopted, since, in the absence of a counterstrategy, the path of least effort is to adopt the externally imposed temporal groupings (Winzenz \& Bower, 1970). By comparison, sentences are endowed with an a priori organization by virtue of their grammatical structure. The words of a sentence are thus syntactically and semantically related. Wilkes and Kennedy (1969;
Kennedy \& Wilkes, 1968) concluded that syntactic structure of sentences, if clearly defined, determines functional groupings, and that retarded latencies occur when retrieval involves crossing major constituent boundaries.

However, Martin (1970) has criticized psycholinguists' total reliance on prescriptive phrase markers to describe the surface structure of sentences without empirically determining whether they correspond to subjective phrase markers. In this spirit, the present study investigated the correspondence between prescriptive and subjective phrase markers.

Specifically, how flexible is the subjective organization of sentences? Can pauses be used to create new constituent boundaries which compete with those arising from syntactic structure? Subjects may, as Martin's (1970) data suggest, attend to the experimenterimposed pauses and adopt the novel organization for memory encoding and retrieval, or subjects might ignore the competing influence in favor of the standard grouping strategy imposed by grammatical structure.

To determine whether syntactic organization has absolute power over the way subjects segment sentences, or whether subjects heed experimenter-imposed pauses when assigning subjective phrase markers, we attempted to manipulate subjective organization by means of a pause between either subject or verb or between the verb and the adjective modifying the object noun. In the first condition, the pause corresponds to where a subject-imposed pause placement would be: Pirate ships-fly black flags. In the second condition, the pause introduces a possibly competing organization: Pirate ships fly-black flags. Thus, although in both cases the pauses were imposed by the experimenter, the latter pause would set up an unnatural grouping format. Comparison of the pattern of retrieval latencies should tell us whether subjective organization is flexible, or if it is solely a function of syntactic organization. 


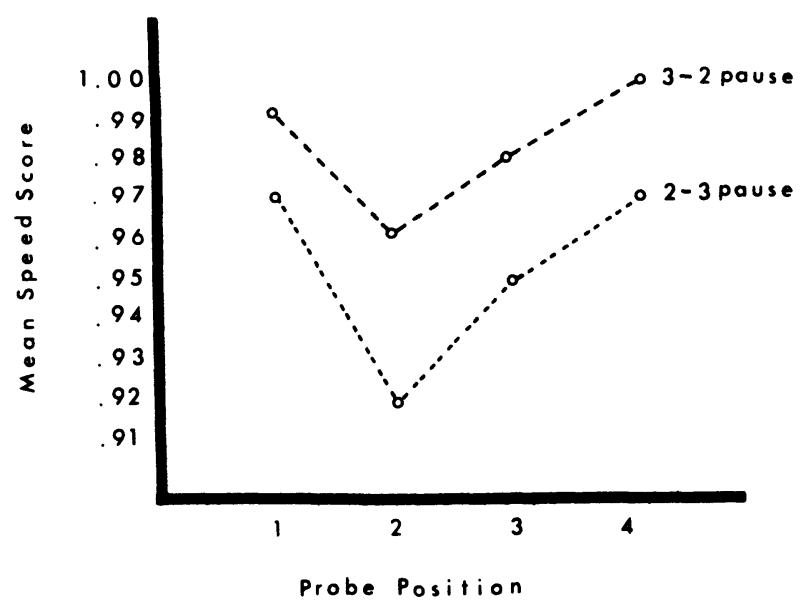

Figure 1. Mean speed scores for Group 1.

\section{METHOD}

\section{Subjects}

Thirty-two Rutgers College students participated in the experiment to fulfill a requirement for their introductory psychology course.

\section{Materials and Design}

The study employed a 2 by 2 by 2 by 4 design, with Groups (2) and Experimenter (2) as between-subject factors, Pause Location ( 2 levels) and Probe Position (4 levels) as withingroups factors, and subjects nested within experimenter.

One hundred and twenty sentences, having the form adjective-noun-verb-adjective-noun $\left(A_{1}-N_{1}-V-A_{2}-N_{2}\right)$, were constructed (e.g., Pirate ships fly black flags.). Of the 120 sentences, 30 were composed solely of one-syllable words, 30 contained only two-syllable words, and 60 were composed of a mixture of one-, two-, and three-syllable words.

All subjects were tested on all 120 conditions and experienced both pause conditions. Group 1 subjects heard Sentences 1-60 with a 2-sec pause introduced between the second and third words in the sentence (2-3 pause); Sentences 61-120 were presented with the pause between Words 3 and 4 in the sentence (3-2 pause). Group 2 subjects heard Sentences $1-60$ with a 3-2 pause and Sentences $61-120$ with a 2-3 pause. Each block of 60 sentences contained an equal random distribution of the three sentence types.

Only the first four words in each sentence were used as probes. Four probe orders were constructed, each composed of successive block randomizations of the four probe positions. Subjects were assigned to probe orders in the order in which they were run. Thus, Subject 1 was tested on Order 1 , Subject 2 on Order 2, etc.

There were 120 test trials for each subject, with each subject tested on only one probe word for each sentence. Subjects in both groups received 15 trials with each type of probe word $\left(A_{1}-N_{1}-V-A_{2}\right)$. Across subjects and groups, all four probes for each sentence were tested equally often.

\section{Procedure}

The sentences were recorded on tape. Approximately $3 \mathrm{sec}$ after the presentation of each sentence, subjects heard the ready signal. At that time, the experimenter pressed a button on the tachistoscope, illuminating the probe word and simultaneously activating a millisecond timer. The subject's response closed a voice-activated relay, terminating stimulus exposure and stopping the timer. A 12 -sec silent interval was allowed between each ready signal and the presentation of the next sentence. Subjects were given a 5 -min rest period between presentations of the two blocks of 60 sentences. Instructions stressed speed and accuracy.

\section{RESULTS}

Error rate was small (4\%) and no error trials were included in the analyses. To reduce the variance of the reaction time score, the reciprocal of each time was computed, yielding a speed score. Mean speed scores were computed for each of the four probe conditions under each pause condition for each subject in both groups. In this way, eight mean speed scores were computed for each subject.

An analysis of variance of speed scores did not find significant main effects of Groups or Pause Condition, but their interaction was significant $[F(1,28)=4.87$, $\mathrm{p}<.05]$. Thus, when the $2-3$ pause condition was presented first, speed scores were slower than for sentences bearing the 3-2 pause. When the 3-2 pause condition was presented first, retrieval was slower than for the following 2-3 pause sentences. Therefore, the pause condition presented second was responded to more rapidly, reflecting a practice effect.

The Experimenter variable was not significant, nor did it interact with any other variable. Probe Position had a significant effect on retrieval latency $[F(3,84)=6.24, p<.01]$. Thus, retrieval latency varied with probe positions within the sentence, consistent with the findings of previous researchers (Wilkes \& Kennedy, 1969).

The predicted Pause Condition by Probe Position interaction only approached significance $[\mathrm{F}(3,84)=$ $1.87, .05<\mathrm{p}<.10]$. Inspection suggested that this interaction depended on the order of the pause conditions. Accordingly, Group 1 and Group 2 were analyzed separately.

Figure 1 presents the mean speed scores for Group 1.

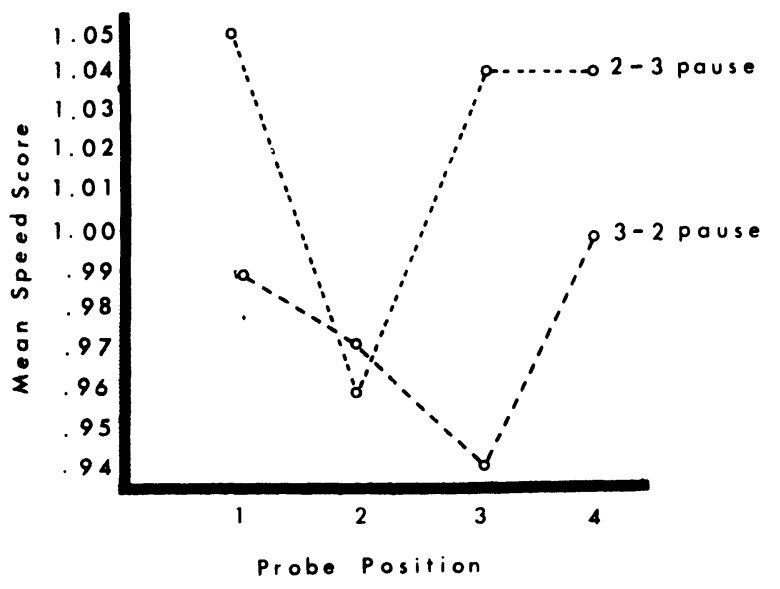

Figure 2. Mean speed scores for Group 2. 
The data indicates faster retrieval across all transitions for the second block of sentences. However, this practice effect was not statistically reliable $[\mathrm{F}(1,15)=1.71$, $\mathrm{p}>.05)]$. Probe Position influenced retrieval latency $[F(3.45)=3.09, \mathrm{p}<.05]$, but its interaction with Pause Condition was not reliable, as the same pattern of latencies was obtained in both pause conditions.

Figure 2 presents the mean speed scores for Group 2, where the 3-2 pause condition was presented first. Probe position was significant, as latency of retrieval varied across transitions $[F(3,45)=4.05, .05>p>.01]$. Once again, subjects tended to respond faster in the second (2-3 condition) block of sentences, but this practice effect was not reliable $[F(1,15)=3.28$, $\mathrm{p}>.05]$.

Inspection of Figure 2 suggests that grouping structure varied with pause condition. The most difficult transition in the 3-2 condition was that between the third and fourth words, while the second transition was the most difficult in the 2-3 condition. The interaction suggested in Figure 2 was not statistically reliable $[F(3,45)=2.70, p>.05]$. However, predictions for retrieval from Probe Positions (transitions) 1 and 4 were not clear, as these transitions fall within a constituent boundary in both pause conditions. The present study was concerned with retrieval across constituent boundaries, and clear-cut predictions were made only for the second and third transitions. Analysis of only Transitions 2 and 3 found the Pause Condition by Probe Position interaction to be significant $[F(1,14)=10.40$, $\mathrm{p}<.01]$. Therefore, retrieval latency from Probe Positions 2 and 3 varied with the pause condition imposed on the sentence. When the lengthened pause was introduced at Transition 2 (2.3 pause), retrieval from Probe Position 2, across a constituent boundary, was slow in comparison to faster retrieval from Probe Position 3, within a constituent. When the lengthened pause was imposed at Transition 3 (3-2 pause), retrieval from Probe Position 3, across a constituent boundary, was slow in comparison to faster retrieval from Probe Position 2, now within a phrase constituent.

\section{DISCUSSION}

The findings give some support to Martin's (1970) argument for flexibility of subjective organization. The grammatical relationships of a sentence do not absolutely determine a subject's grouping strategy. Pauses can influence organization, but the influence is not great and is easily eliminated by prior experience with the "standard" organization.

A similar organizational stubbornness can be produced in subjects even with unrelated lists. Winzenz and Bower (1970) investigated the relationship between accumulative learning over successive repetitions of a digit series and the identity of codings of recurrences of the series. Their previous studies (Bower \& Winzenz, 1969) had shown no accumulative learning if a series is regrouped in new ways each time it recurs. The later study pretrained subjects to recode each 12-digit series into a standard format. With this recoding strategy, subjects did learn despite the series being regrouped differently at each occurrence. Subjects' recall units were determined by the groupings they imposed on the series, rather than by the experimenter's pausemarked groupings. Different regroupings of a string by the experimenter were translated into the same subjective format.

The parallel between the present results and those of Winzenz and Bower (1970) are obvious, the primary difference lying in the origin of the grouping strategies. With sentences, grouping strategies are suggested by the subject's implicit grammar. The pretraining given by Winzenz and Bower can be thought of as teaching subjects a grammar for parsing strings of digits. In both cases, subjects appear to have a strong predilection for persisting with their "grammatical" analysis (at least, under the respective constraints of the two experimental paradigms).

Of course, the parallel is not exact, for digit "grammars" are obviously not as restrictive as natural language grammars. The asymmetry is evident in the present data: Initial experience with the canonical grouping persisted even when a contrasting grouping was introduced; however, initial experience with a nonstandard grouping did not persist. The nonstandard grouping was readily abandoned when a standard grouping was introduced. Such an asymmetry would not be observed with digit series.

In summary, while digit series are more flexible than sentences, sentences have more flexibility than is suggested by a prescriptive phrase marker analysis. However, this flexibility is more easily destroyed in the sentences than in the digit series. This suggests that, for the probe continuation task, the primary difference between lists and sentences is one of degree rather than kind.

\section{REFERENCES}

Bower, G. H., \& Winzenz, D. Group structure, coding, and memory for digit series. Journal of Experimental Psychology, 1969, 80(2, Part 2).

Butterfield, E. C., \& Belmont, J. M. Relations of storage and retrieval strategies as short-term memory processes. Journal of Experimental Psychology, 1971, 89, 319-328.

DeRosa, D. V., \& Baumgarte, R. Probe digit recall of items from temporally organized memory sets. Journal of Experimental Psychology, 1971, 91, 154-158.

Kennedy, R. A., \& Wilkes, A. L. Response times at different positions within a sentence. Quarterly Journal of Experimental Psychology, 1968, 20, 390-394.

Martin, E. Towards an analysis of subjective phrase structure. Psychological Bulletin, 1970, 74, 153-166.

McLeAN, R. S., \& GREGG, L. W. Effects of induced chunking on temporal aspects of serial recitation. Joumal of Experimental Psychology, 1967, 74(4, Part 1).

STERnberg, S. Memory scanning: Mental processes revealed by reaction time experiments. American Scientist, 1969, 57, 421-425.

WILKES, A. L., \& KENNEDY, R. A. Relationship between pausing and retrieval latency in sentences of varying grammatical form. Journal of Experimental Psychology, 1969, 79, 241-245.

Wilkes, A. L., \& KenNedy, R. A. The relative accessibility of list items within different pause-defined groups. Journal of Verbal Learning and Verbal Behavior, 1970, 9, 197-201.

Winzenz, D., \& Bower, G. H. Subject-imposed coding and memory for digit series. Journal of Experimental Psychology, $1970,83,52-56$.

(Received for publication July 6, 1976.) 